\title{
THE STRUCTURE OF COPPER RUBBED ON EMERY PAPER AT DIFFERENT TEMPERATURES
}

\author{
J. H. DAUTZENBERG and J. A. B. VAN DIJCK \\ Division of Production Technology, Eindhoven University of Technology, Den Dolech 2, \\ Postbus 513, 5600 MB Eindhoven (The Netherlands)
}

(Received May 15, 1981; in revised form October 2, 1981)

Summary

The physical process involved in the rubbing of copper on emery paper resembles the dry sliding wear of copper against SAE 1045 steel. In both cases the top layer of the contact surface, the thickness of which is different for the two processes, is heavily deformed. The heavily deformed material can recrystallize dynamically in accordance with the Zener-Hollomon parameter. When copper undergoes this process at room temperature at high strain rate and high strain, very small grains are formed in the top layer of the rubbed surface. At liquid nitrogen temperature the copper partially recrystallizes dynamically in accordance with the Zener-Hollomon parameter. The occurrence of dynamic recrystallization results in a surface layer with very low residual stresses, high toughness and high hardness. These qualities are desirable in manufactured surfaces.

\section{Introduction}

The physical process that occurs when a metal is rubbed on abrasive paper has often been compared with the cutting process in turning. In turning, the metal deforms plastically in two regions (Fig. 1), the primary shear zone and the secondary deformation zone. The natural strain in the primary shear zone is about a factor of $\mathbf{5}$ greater than that in the forming process, and the strain in the secondary shear zone is even higher [1]. The volume of the secondary zone is relatively small compared with the chip volume. Therefore the energy for the process in this zone is relatively small (about $30 \%$ of the total energy required for the cutting process).

The process in the secondary zone is assumed to be comparable with the dry sliding wear process and with the rubbing process on emery paper. The aim of this work was to investigate this assumption. If it is true, very highly deformed or dynamically recrystallized material will be present in the rubbed contact zone of the metal. Material which has undergone dynamic recrystallization has a low residual stress, a very fine structure, a 


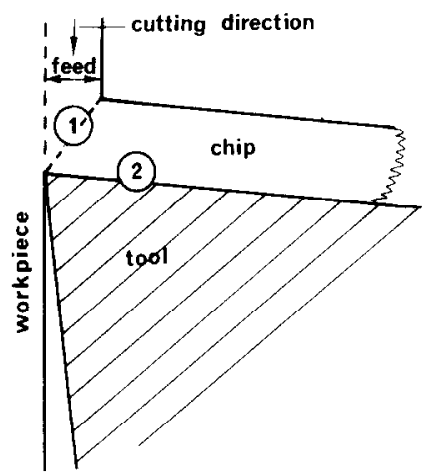

Fig. 1. Schematic two-dimensional representation of the cutting process in turning: 1 , primary shear zone; 2 , secondary deformation zone.

high flow stress and a high toughness, i.e. a surface layer with very good mechanical properties which are often desired in production. The effects of dynamic recrystallization also explain the weak texture, i.e. the orientation distribution of the metal grains, of a rubbed surface layer [2]. The occurrence of this process increases in importance with demands for improved surface quality in metal working production.

There is much more information available concerning the initiation of dynamic recrystallization under normal conditions than under rubbing conditions [3]. The kinetics have been described [4 - 7] and the occurrence of the process in a metal is determined by the strain rate, the temperature and the strain level. Recrystallization may be either controlled or uncontrolled.

We have estimated the natural strain for rubbing copper on emery paper. The determination of the necessary material constants is described and carried out in Appendix A. The occurrence of dynamic recrystallization as a function of the different parameters is briefly summarized. The experimental results are discussed, and it is concluded that the physical process involved in the rubbing of copper on emery paper is the same as that involved in dry sliding wear.

\section{Computation of the natural strain}

If it is assumed that the specific energy $E_{\mathrm{sp}}$ for rubbing of a metal on emery paper is that required for the homogeneous plastic deformation of the metal, then

$$
E_{\mathrm{sp}}=\int_{0}^{\bar{\epsilon}} \sigma \mathrm{d} \bar{\epsilon}
$$

where $\bar{\sigma}$ is the effective stress defined by

$$
2 \bar{\sigma}^{2}=\left(\sigma_{11}-\sigma_{22}\right)^{2}+\left(\sigma_{22}-\sigma_{33}\right)^{2}+\left(\sigma_{33}-\sigma_{11}\right)^{2}+6\left(\sigma_{12}{ }^{2}+\sigma_{13}{ }^{2}+\sigma_{23}{ }^{2}\right)
$$


and the $\sigma_{i j}$ are the components of the stress tensor, $\bar{\epsilon}$ is the effective natural strain defined by

$$
\begin{aligned}
\dot{\vec{\epsilon}}^{2}= & \frac{4}{9}\left[\frac{1}{2}\left\{\left(\dot{\epsilon}_{11}-\dot{\epsilon}_{22}\right)^{2}+\left(\dot{\epsilon}_{22}-\dot{\epsilon}_{33}\right)^{2}+\left(\dot{\epsilon}_{33}-\dot{\epsilon}_{11}\right)^{2}\right\}+\right. \\
& \left.+3\left(\dot{\epsilon}_{12}^{2}+\dot{\epsilon}_{13}{ }^{2}+\dot{\epsilon}_{23}^{2}\right)\right]^{1 / 2}
\end{aligned}
$$

and the $\dot{\epsilon}_{i j}$ are the components of the incremental strain velocity tensor, and $\bar{\epsilon}_{\mathrm{m}}$ is the maximum natural strain. By using the Nadai relation

$$
\bar{\sigma}=C \bar{\epsilon}^{n}
$$

where $C$ is the specific stress and $n$ is the strain-hardening exponent we obtain from relation (1)

$$
\bar{\epsilon}_{\mathrm{m}}=\left\{\frac{E_{\mathrm{sp}}(n+1)}{C}\right\}^{1 /(n+1)}
$$

The specific energy $E_{\mathrm{sp}}$ for rubbing can be derived by measuring the frictional force during rubbing and determining the mass loss of the test pieces for a given distance.

Table 1 gives these values as a function of the grain thickness of the emery paper. With $n=0.28$ and $C=400 \mathrm{~N} \mathrm{~mm}^{-2}$ (see Appendix A), the effective strain can be derived as a function of the grade of the emery paper. Table 1 shows that the specific energy increases with decreasing grain size of the emery paper. This is in agreement with turning where the specific energy increases with decreasing feed.

TABLE 1

Test results for copper rubbed on different grades of emery paper

\begin{tabular}{llllll}
\hline Grade of emery paper & 150 & 240 & 320 & 400 & 600 \\
& & & & & \\
Grain diameter $(\mu \mathrm{m})$ & $88-105$ & $45-53$ & $31-37$ & $27-31$ & $18 \cdot 22$ \\
Mass loss $(\mathrm{mg})$ & 97.7 & 71.7 & 78.8 & 71.7 & 52.9 \\
Distance $(\mathrm{m})$ & 5.25 & 5.25 & 5.25 & 5.25 & 5.25 \\
$E_{\text {sp }}\left(\mathrm{J} \mathrm{mm} \mathbf{~ m}^{-3}\right)$ & 17.6 & 20.7 & 21.1 & 22.4 & 22.8 \\
$\bar{\epsilon}_{\mathrm{m}}$ & 23.3 & 26.5 & 26.9 & 28.2 & 28.5 \\
Friction force $(\mathrm{N})$ & 36.5 & 31.6 & 35.3 & 34.1 & 25.6 \\
\hline
\end{tabular}

Dimensions of the test pieces, $25 \mathrm{~mm} \times 15 \mathrm{~mm} \times 5 \mathrm{~mm}$; dimensions of the rubbing surface, $25 \mathrm{~mm} \times 15 \mathrm{~mm}$; rubbing velocity, $0.2 \mathrm{~m} \mathrm{~s}^{-1}$.

\section{Dynamic recrystallization}

Dynamic recrystallization is the process by which the material recrystallizes during deformation under certain conditions. The theory of this process 
has been discussed in detail elsewhere $[4,5]$. This recrystallization process is local, and can occur once or several times depending on the test conditions. The principal sites for dynamically recrystallized grains are the pre-existing grain boundaries $[4,6]$. This local behaviour in hot-working experiments at low strains and low strain rates can be expressed by the Avrami relation

$$
X=1-\exp \left(-k t^{a}\right)
$$

where $X$ is the fraction of the microstructure consisting of dynamically recrystallized grains, $k$ and $a$ are constants and $t$ is the time from the start of the reaction. The constants $k$ and $a$ are determined not only by external factors which can affect the recrystallization (temperature, grain thickness and strain rate) but also by the reaction mode, i.e. the nature of the nucleation sites and the time dependences of the nucleation rate and the growth rate. The value of the constant $k$ can be influenced by the grain thickness. The occurrence of dynamic recrystallization can be influenced by the temperature, the strain rate and the effective stress [7]. These dependences $[4,5]$ can be represented by the Zener-Hollomon parameter which is defined by

$$
Z=\dot{\bar{\epsilon}} \exp (Q / R T)
$$

where $Q$ is the activation energy and $R$ and $T$ have their usual meanings.

When the Zener-Hollomon parameter is plotted on a double logarithmic plot, a linear relation is obtained with the critical stress $\bar{\sigma}_{\mathrm{c}}$, which is equivalent to the starting stress of the process; a high value of $Z$ implies a high value of $\bar{\sigma}_{c}$. From eqn. (4), a high value of $\bar{\sigma}$ implies a high value of the effective strain (for values of $d \ln \bar{\sigma}_{c} / d \ln Z$ see ref. 5). A consequence of this behaviour is that a material with large grains needs a higher degree of deformation than the same material with smaller grains under the same test conditions (see relation (8)).

The size of the dynamically recrystallized grains increases monotonically with decreasing $\bar{\sigma}_{\mathbf{c}}$ and can be represented by the phenomenological HallPetch relation

$$
\bar{\sigma}_{\mathrm{c}}=\bar{\sigma}_{0}+A d^{-\imath}
$$

where $\bar{\sigma}_{0}, A$ and $b$ are empirical constants. The value of $b$ is in the range $0.5-0.8[5]$.

An example of the effect of dynamic recrystallization on a stress-strain curve for different strain rates is shown in Fig. 2. Figure 2 shows flow curves of a plain $0.25 \% \mathrm{C}$ steel in the austenitic state (f.c.c.) at $1100{ }^{\circ} \mathrm{C}$. At high strain rates they show the typical shape for dynamic recrystallization: strain hardening to peak stress followed by work softening to a steady state level. The work softening is caused by the onset of dynamic recrystallization and the stable flow results from continuous dynamic recrystallization. At low strain rates, the flow curve is periodic owing to recurrent cycles of recrystallization. 


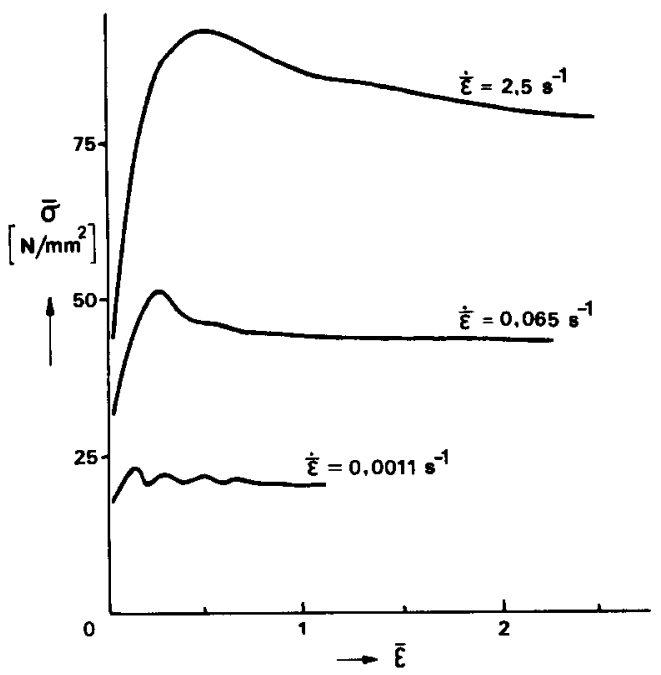

Fig. 2. The effect of dynamic recrystallization on the stress-strain curves obtained at different strain rates for a plain $0.25 \% \mathrm{C}$ steel in the austenitic state at $1100^{\circ} \mathrm{C}$ [3].

\section{Experimental details}

All the tests were performed on copper, which was always rubbed in one direction by hand. Two types of specimen were used.

(1) Specimens of type 1 were used to determine $E_{\text {sp }}$. They were of dimensions $25 \mathrm{~mm} \times 15 \mathrm{~mm} \times 5 \mathrm{~mm}$, and the rubbed surface was of area $25 \mathrm{~mm} \times 15 \mathrm{~mm}$. They were not vacuum annealed. In order to measure the frictional force, the emery paper was stuck to a table placed on springs. The frictional force was determined from the bending of the springs which was measured with strain gauges. The frictional energy computed from the frictional force and the total rubbing distance together with the weight loss of the samples gives $E_{\text {sp }}$ (Table 1).

(2) Specimens of type 2 were of dimensions $20 \mathrm{~mm} \times 10 \mathrm{~mm} \times 0.1 \mathrm{~mm}$, and the rubbing surface was of area $20 \mathrm{~mm} \times 10 \mathrm{~mm}$. These samples were vacuum annealed for $3 \mathrm{~h}$ at $750^{\circ} \mathrm{C}$. After rubbing on emery paper they were used for making thin foils. The rubbed surface was covered by plastic tape so that the thinning process only occurred from the opposite side. The thinning process was stopped automatically as soon as a hole appeared in the specimen. The thinning process has been fully described elsewhere [8]. The low temperature rubbing tests were performed in an open box totally filled with liquid nitrogen. The emery paper was stuck to a piece of metal lying in the open box. The sample was clamped in a pair of tongs for rubbing on the emery paper.

The electron micrographs were prepared using a Philips EM 200 electron microscope. 


\section{Results}

The electron micrograph of a thin foil of the surface of copper rubbed on grade 240 emery paper reproduced in Fig. 3 shows dynamically recrystallized grains and heavily deformed material. A number of electron micrographs made under the same conditions show that most of the copper is recrystallized, i.e. $X$ is almost unity, in relation (6). If it is assumed that the grains have an elliptical form and axes $a$ and $b$ are both measured, the average surface area $\pi a b / 4$ is $0.0216 \mu \mathrm{m}^{2}$. The distribution of these values is shown in Fig. 4 as a relative cumulative frequency diagram of the logarithmic value of the grain surface. A straight line means that the distribution of the quantity described is log-normal. The number of measurements was 101.

The recrystallized grains in the middle of the rubbing track appear to be smaller than those at the boundaries, but the number of measurements was too small for confirmation. Figure 5 shows a typical electron micrograph of a thin foil of the copper surface rubbed on 320 emery paper in liquid nitrogen. In all micrographs obtained from this foil the number of recrystallized grains is small. In most cases heavily deformed rather than recrystallized copper was found. Measurements made under the same conditions as in Fig. 3 gave an average grain surface area of $0.0065 \mu \mathrm{m}^{2}$, i.e. appreciably different from the grain surface area of copper rubbed at room temperature. The distribution of the grain surface is also presented in Fig: 4. A comparison of both distributions in Fig. 4 shows that the distributions are the same but the averages are different. This supports the assumption that

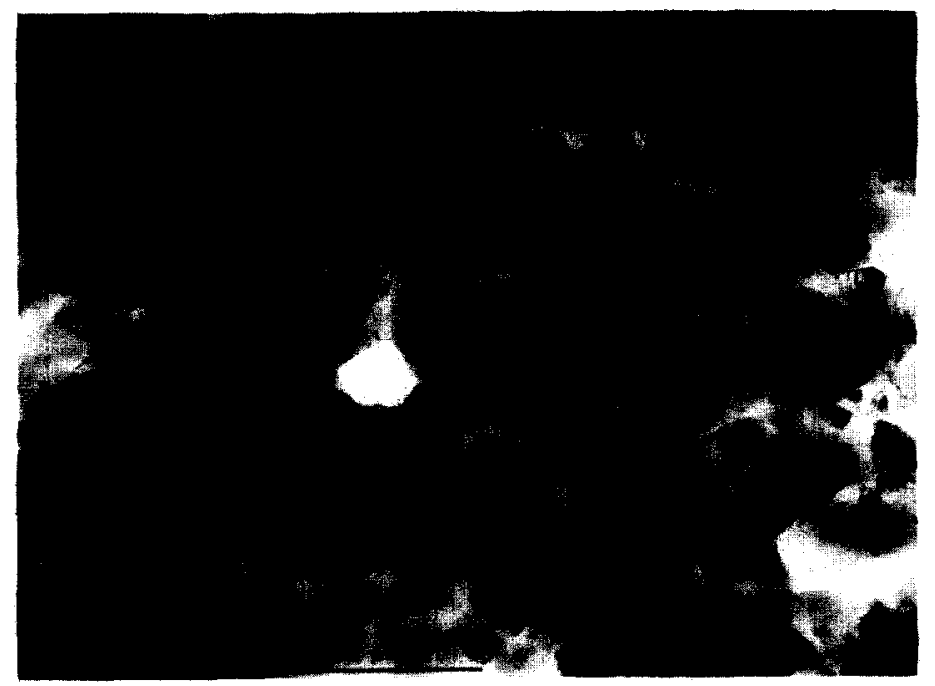

Fig. 3. Electron micrograph of a thin foil of the surface of copper rubbed on emery paper 240. 


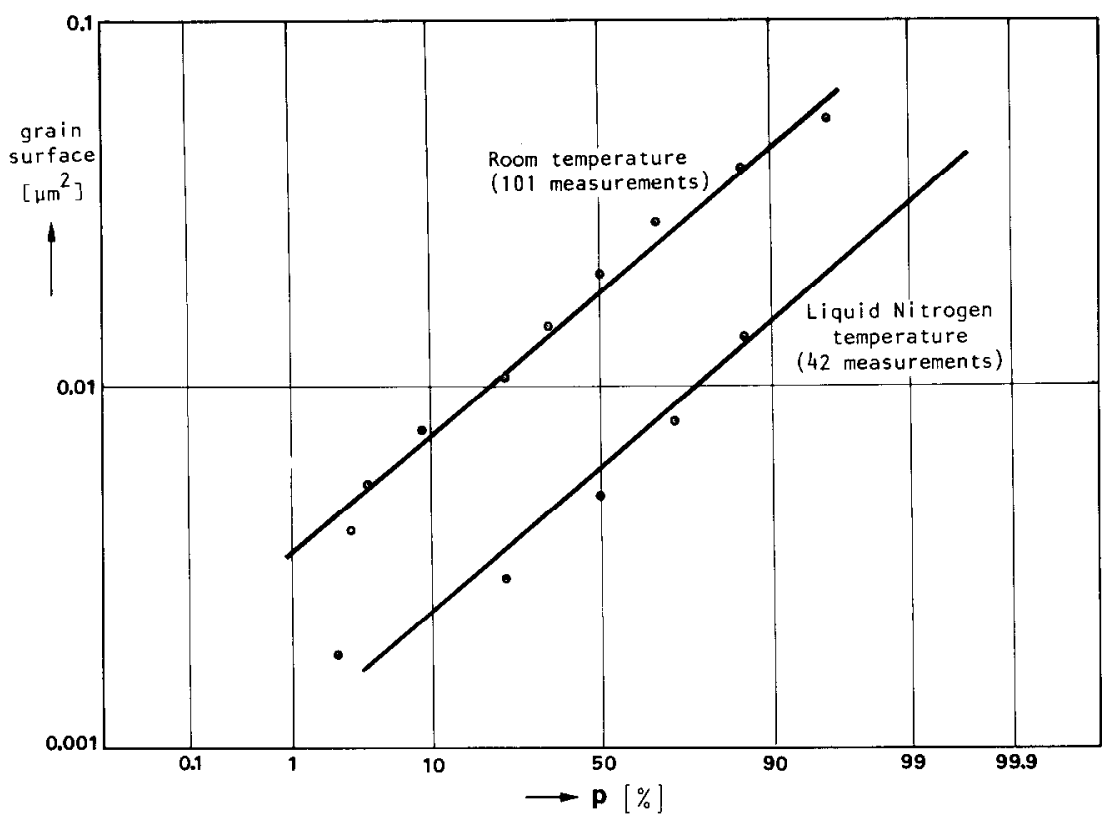

Fig. 4. The log-normal distribution of the grain surface of a copper sample rubbed on emery paper.

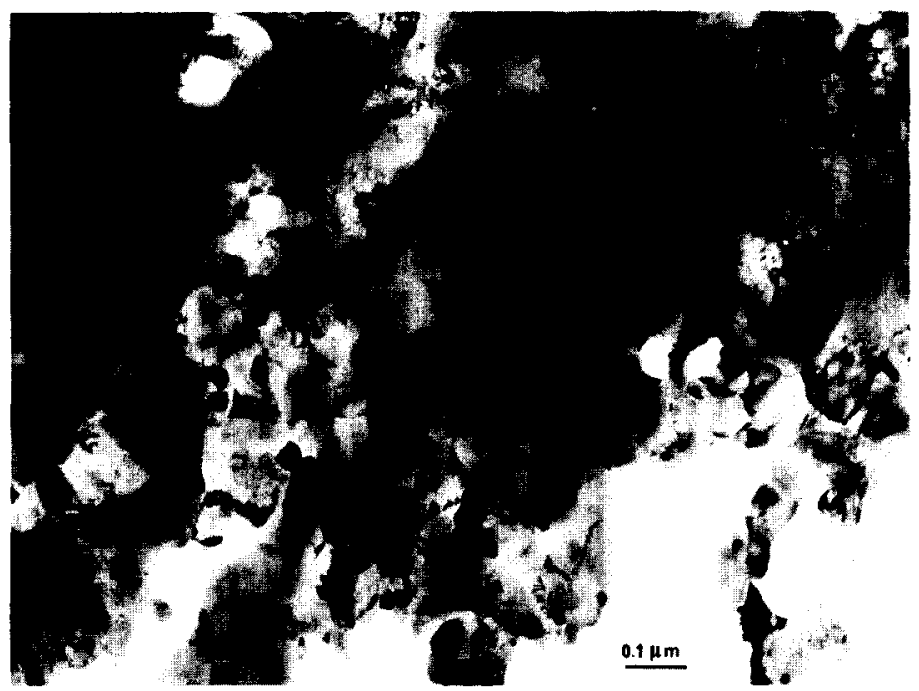

Fig. 5. Electron micrograph of a thin foil of the surface of copper rubbed on emery paper 320 at liquid nitrogen temperature.

the process is the same. The electron diffraction pattern (Fig. 6) of the area shown in Fig. 5 confirms that the grains in Fig. 5 are real. Figure 6 is from an area of $3 \mu \mathrm{m}^{2}$. 


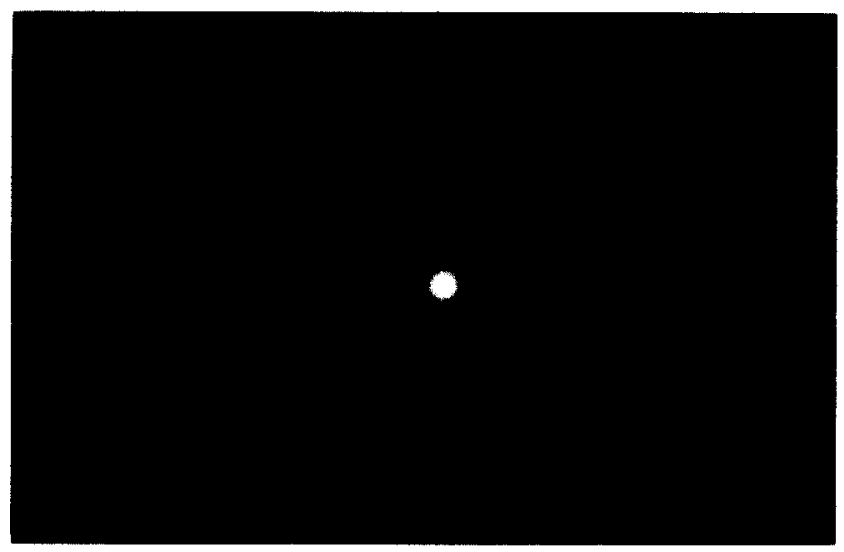

Fig. 6. Electron diffraction pattern of a part of the surface shown in Fig. 5. This pattern was obtained from an area of $3 \mu \mathrm{m}^{2}$

\section{Discussion}

The almost total recrystallization of copper rubbed at room temperature demonstrates that the effective stress was just high enough for the test conditions, quantified by the Zener-Hollomon parameter, to allow dynamic recrystallization of copper. When the copper is rubbed under liquid nitrogen under the same test conditions, a higher Zener-Hollomon parameter $Z$ is obtained from eqn. (6) which implies a higher critical stress for dynamic recrystallization. If the stresses necessary for rubbing at room temperature and at liquid nitrogen temperature are equal, then no recrystallization occurs at liquid nitrogen temperature. It can be concluded from electron micrographs of thin foils of rubbed copper at this temperature that only local dynamic recrystallization takes place, which is understandable. A decrease in the temperature produces an increase in the stress for a given strain. This increase is smaller than that caused by increasing $Z$ so that only a small part of the volume recrystallizes dynamically at liquid nitrogen temperature. The local and temperature-dependent behaviour shown in the electron micrographs of rubbed copper supports the hypothesis that the operative process is dynamic recrystallization.

At higher values of $Z$ a higher critical stress for dynamic recrystallization is required, and hence, from relation (8), the grain diameter or the grain surface area will be smaller. Such behaviour is characteristic of dynamic recrystallization. Figure 4 confirms this behaviour, and hence provides additional support for the occurrence of dynamic recrystallization.

If it is assumed that the electron micrographs are arbitrary cross sections of the rubbed copper and that the grains are balls of the same diameter $D$, we obtain

$$
D=(6 \widetilde{O} / \pi)^{1 / 2}
$$


where $\bar{O}$ is the average of the measured elliptical surface of the grains. These grains were assumed to be elliptical rather than circular only in order to account for the different lengths of the two perpendicular axes in the grains. For $\bar{O}$ values of 0.0216 and 0.0065 at room temperature and at liquid nitrogen temperature respectively, relation (9) gives the grain diameter of rubbed copper as $0.2 \mu \mathrm{m}$ and $0.1 \mu \mathrm{m}$ respectively. These values agree well with grain diameters measured in the dry sliding wear of copper against SAE 1045 steel [2].

Processes involving dry sliding friction such as dry sliding wear [2], rubbing and cutting [1] produce heavily deformed or dynamically recrystallized material with grain diameters of the same order of magnitude in the contact surface. All these processes appear to be the same. As a result of dynamic recrystallization the hardness of the upper layer of these materials will be higher than that of the static recrystallized material but lower than that of the subsurface material. This may be the reason for the concentration of deformation in this layer. The top layer also has low residual stresses and high toughness. These phenomena are usually found in ground surfaces [9] and can be explained by dynamic recrystallization. If it is possible to control the dynamic recrystallization process, it could be important in metalworking production since it would allow a surface layer with different qualities to the bulk material to be produced.

When using eqn. (5) to calculate $\bar{\epsilon}$ for the rubbing process it is assumed that the values of $C$ and $n$, which are determined at room temperature and for strains which are large for tensile tests but small for rubbing tests, are also valid under the conditions used here, i.e. high strains and high strain rates. The effect of dynamic recrystallization on the stress-strain curve is neglected. When both these effects are taken into account an estimation of the variation in $\vec{\epsilon}$ shows that their influence on its value is relatively small.

It is assumed in the derivation of relation (5) that $\bar{\epsilon}$ is homogeneous in the chip. It is more likely that, as with cutting, the largest strain is in the friction zone, i.e. the secondary shear zone in the cutting process. This means that the deformation energy in the secondary shear zone is higher than that in the rest of the chip. This is supported by the increase in $E_{\mathrm{sp}}$ with decreasing chip thickness.

The determination of the effective strain by measurements of the grain diameter as proposed by Dautzenberg and Zaat [10] is not applicable to dynamically recrystallized material.

The computed effective strains of the upper part of the rubbed surface layer and of the rubbed material appear to be the same. This may not be strictly true, but suggests that they are of the same order of magnitude.

\section{Conclusions}

When copper is rubbed on emery paper the grains in the top layer are heavily deformed and new very small grains can be created during this 
process. The creation of these grains can be explained by a dynamic recrystallization process. The temperature dependence and the quantity of material correspond to a first approximation to dynamic recrystallization behaviour. Under normal conditions this behaviour can be described by the Zener-Hollomon and the Avrami relations. However, the rubbing test conditions deviate substantially. This process can explain the low residual stresses and relatively high hardness compared with the static recrystallized material in the top layer. The friction in dry sliding wear may be the same physical process as that involved in rubbing.

\section{References}

1 J. H. Dautzenberg, J. A. B. van Dijck and A. C. H. van der Wolf, On some aspects of dynamic recrystallization in several manufacturing processes, CIRP Note STC, C Subgroup Fundamentals, Davos, August 1979 (available to CIRP members or on request).

2 R. J. Zaalberg, J. H. Dautzenberg and J. A. Klostermann, Deformation and recrystallization in copper and other metals formed by dry sliding against steel. In Proc. 5 th Int. Conf. on Textures of Materials, Vol. 2, Springer, Berlin, 1978, pp. $379-388$.

3 R. J. Arsenault, Treatise on Materials Science and Technology, Vol. 6, Plastic Deformation of Materials, Academic Press, New York, 1975.

4 W. Roberts, H. Boden and B. Ahlborn, Dynamic recrystallization kinetics, Met. Sci., 13 (1979) 195 - 203.

5 W. Roberts and B. Ahlborn, A nucleation criterion for dynamic recrystallization during hot working, Metallurgica, 26 (1978) $801-813$.

6 G. Gottstein, D. Zabardjadi and H. Mecking, Dynamic recrystallization in tension deformed copper single crystals, Met. Sci., 13 (1979) $223-227$.

7 P. J. T. Stuitje und F. Gottstein, Dynamische Rekristallisation in zugverformten Silbereinkristallen, Z. Metallkd., 71 (1980) $279-285$.

8 J. A. B. van Dijck, The direct observation in the transmission electron microscope of the heavily deformed surface layer of a copper pin after dry sliding against a steel ring, Wear, 42 (1977) $109-117$.

9 G. Bellows, Low stress grinding for quality production, Publ. MCD-78-103, 1978 (Machinability Data Center, Cincinnati, $\mathrm{OH}$ ).

10 J. H. Dautzenberg and J. H. Zaat, Quantitative determination of deformation by sliding wear, Wear, 23 (1973) 9 - 19.

\section{Appendix A}

\section{Determination of the material constants $C$ and $n$}

The material constants $C$ and $n$ are usually determined using the tensile test. In order to extrapolate the effective strain area to higher values (the tensile test is normally constrained to homogeneous deformation), the approximation method described by Bridgman [A1] is used. This method enables the stress and strain in necking to be calculated.

Under these conditions the effective stress is [A1]

$$
\bar{\sigma}=C_{\mathrm{B}} \frac{F}{A}
$$

where $F$ is the tensile force, $A$ is the minimum cross-sectional area of the test bar and 


$$
C_{\mathrm{B}}=\frac{1}{(1+2 R / a) \ln (1+a / 2 R)}
$$

where $a$ is the smallest radius of the test bar (Fig. A1) and $R$ is the minimum necking radius of the test bar (Fig. A1). From relation (3), the effective strain in the necking area is

$$
\bar{\epsilon}=2 \ln \left(a_{0} / a\right)
$$

where $2 a$ is the diameter of the undeformed test bar.

The two quantities $\bar{\sigma}$ and $\bar{\epsilon}$ are related to each other by the Nadai relation

$$
\bar{\sigma}=C \bar{\epsilon}^{n}
$$

$C$ and $n$ are determined by plotting these values on a double logarithmic scale on the $y$ and the $x$ axes. The strain-hardening exponent $n$ is given by the gradient of the line of closest fit, and $C$ is the value of $\bar{\sigma}$ at which the extrapolated line cuts the line $\bar{\epsilon}=1$.

Figure A2 shows a log-log representation of the experimentally determined relation between $\bar{\sigma}$ and $\bar{\epsilon}$ for copper, and it can be seen that there are two areas with different values of $C$ and $n$. Because of the high strains involved in rubbing, the values of $\bar{\sigma}$ and $\bar{\epsilon}$ in the second area are taken, i.e. $C=400 \mathrm{~N} \mathrm{~mm}^{-2}$ and $n=0.28$.
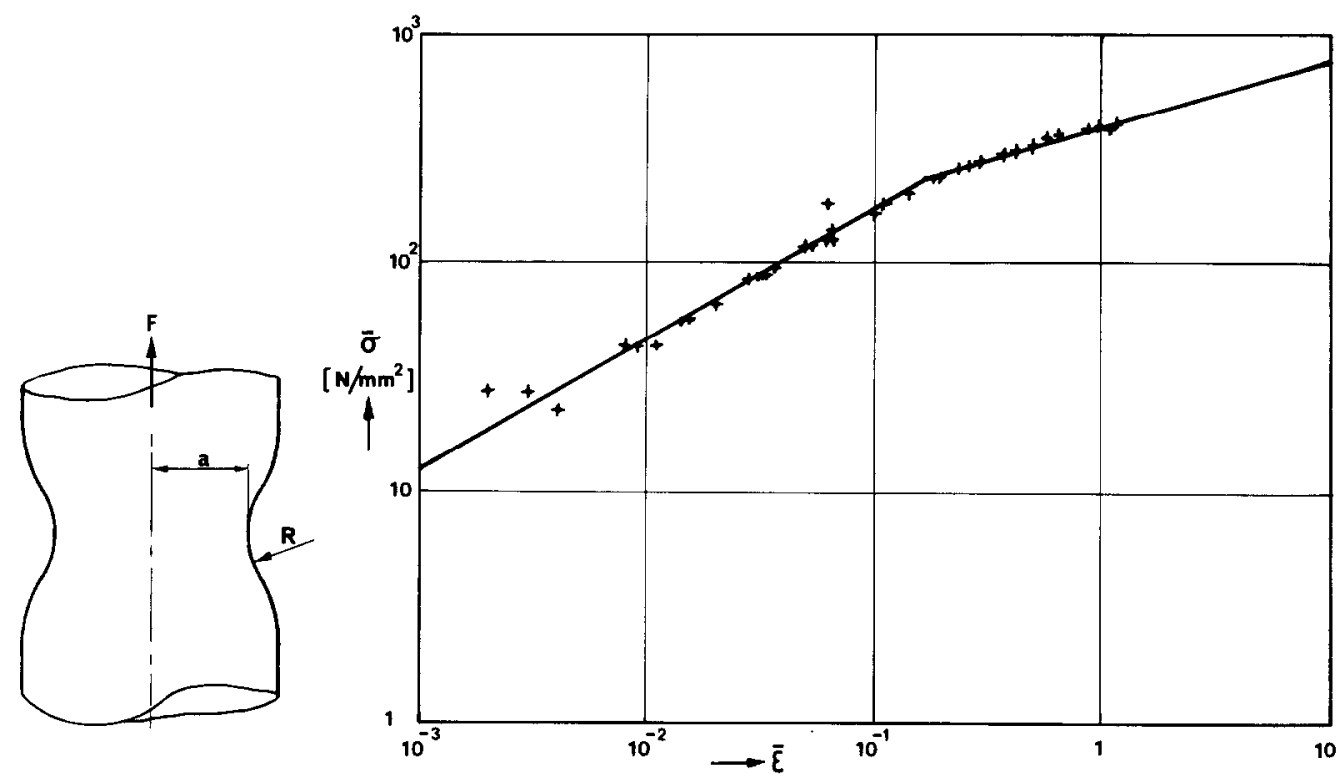

Fig. A1. The necking of a tensile bar with its dimensions.

Fig. A2. Experimental representation of the $\bar{\sigma}-\bar{\epsilon}$ relation derived from the tensile test for copper.

Reference for Appendix A

A1 P. W. Bridgman, Studies in Large Plastic Flow and Fracture, McGraw-Hill, New York, 1952 . 